\title{
Homogeneous protein analysis by magnetic core-shell nanorod probes
}

Stefan Schrittwieser, Beatriz Pelaz, Wolfgang J Parak, Sergio Lentijo-Mozo, Katerina

Soulantica, Jan Dieckhoff, Frank Ludwig, Thomas Altantzis, Sara Bals, and Joerg Schotter

ACS Appl. Mater. Interfaces, Just Accepted Manuscript • DOI: 10.1021/acsami.5b11925 • Publication Date (Web): 29 Mar 2016

Downloaded from http://pubs.acs.org on April 3, 2016

\section{Just Accepted}

"Just Accepted" manuscripts have been peer-reviewed and accepted for publication. They are posted online prior to technical editing, formatting for publication and author proofing. The American Chemical Society provides "Just Accepted" as a free service to the research community to expedite the dissemination of scientific material as soon as possible after acceptance. "Just Accepted" manuscripts appear in full in PDF format accompanied by an HTML abstract. "Just Accepted" manuscripts have been fully peer reviewed, but should not be considered the official version of record. They are accessible to all readers and citable by the Digital Object Identifier (DOI®). "Just Accepted" is an optional service offered to authors. Therefore, the "Just Accepted" Web site may not include all articles that will be published in the journal. After a manuscript is technically edited and formatted, it will be removed from the "Just Accepted" Web site and published as an ASAP article. Note that technical editing may introduce minor changes to the manuscript text and/or graphics which could affect content, and all legal disclaimers and ethical guidelines that apply to the journal pertain. ACS cannot be held responsible for errors or consequences arising from the use of information contained in these "Just Accepted" manuscripts. 


\title{
Homogeneous protein analysis by magnetic core- shell nanorod probes
}

\author{
Stefan Schrittwieser ${ }^{*}$, Beatriz Pelaz ${ }^{2}$, Wolfgang J. Parak ${ }^{2,3}$, Sergio Lentijo-Mozo ${ }^{\dagger 4}$, Katerina \\ Soulantica ${ }^{4}$, Jan Dieckhoff ${ }^{+5}$, Frank Ludwig ${ }^{5}$, Thomas Altantzis ${ }^{6}$, Sara Bals $^{6}$, and Joerg Schotter ${ }^{1}$ \\ ${ }^{1}$ Molecular Diagnostics, AIT Austrian Institute of Technology, Vienna, Austria \\ ${ }^{2}$ Fachbereich Physik, Philipps-Universität Marburg, Marburg, Germany \\ ${ }^{3}$ CIC Biomagune, San Sebastian, Spain \\ ${ }^{4}$ Laboratoire de Physique et Chimie des Nano-objets (LPCNO), Université de Toulouse; INSA, \\ UPS, CNRS, Toulouse, France \\ ${ }^{5}$ Institute of Electrical Measurement and Fundamental Electrical Engineering, TU \\ Braunschweig, Braunschweig, Germany \\ ${ }^{6}$ Electron Microscopy for Materials Research (EMAT), University of Antwerp, Antwerp, \\ Belgium \\ KEYWORDS: magnetic nanoparticle, core-shell nanorod, surface functionalization, \\ homogeneous measurement, anisotropic polarizability, protein analysis, dispersion stability
}




\begin{abstract}
Studying protein interactions is of vital importance both to fundamental biology research and to medical applications. Here, we report on the experimental proof of a universally applicable labelfree homogeneous platform for rapid protein analysis. It is based on optically detecting changes in the rotational dynamics of magnetically agitated core-shell nanorods upon their specific interaction with proteins. By adjusting the excitation frequency, we are able to optimize the measurement signal for each analyte protein size. In addition, due to the locking of the optical signal to the magnetic excitation frequency, background signals are suppressed, thus allowing exclusive studies of processes at the nanoprobe surface only. We study target proteins (soluble domain of the human epidermal growth factor receptor 2 - sHER2) specifically binding to antibodies (trastuzumab) immobilized on the surface of our nanoprobes and demonstrate direct deduction of their respective sizes. Additionally, we examine the dependence of our measurement signal on the concentration of the analyte protein, and deduce a minimally detectable sHER2 concentration of 440 pM. For our homogeneous measurement platform, good dispersion stability of the applied nanoprobes under physiological conditions is of vital importance. To that end, we support our measurement data by theoretical modeling of the total particle-particle interaction energies. The successful implementation of our platform offers scope for applications in biomarker-based diagnostics as well as for answering basic biology questions.
\end{abstract}

\title{
1. Introduction
}

The study of specific proteins within a sample solution is essential in fundamental biology research, e.g. for investigating protein-protein or protein-DNA interactions ${ }^{1,2}$, but also presents the key to medical applications such as molecular diagnostics ${ }^{3,4}$. Studying molecular interactions directly in solution is accomplished by homogeneous measurement principles, which, in contrast 
to heterogeneous techniques, do not rely on diffusion to and interaction with binding partners immobilized on macroscopic surfaces. To date, a number of homogenous techniques have been established for specific areas of application, such as fluorescence correlation spectroscopy ${ }^{5}$, fluorescence polarization detection ${ }^{6}$ or thermophoresis ${ }^{7}$. Homogeneous methods are especially relevant for the development of in-vivo techniques, where direct investigation of processes in live cells is of key importance. ${ }^{8}$ To that end, numerous types of nanoparticles have been developed. Such nanomaterials allow tailoring of physical properties, which can be utilized, for example, for fluorescence-based intracellular observation ${ }^{9}$, magnetic drug targeting ${ }^{10}$ or photothermal cancer therapy $^{10}$.

In this article, we present the experimental proof-of-principle of a universally applicable homogeneous platform for molecular interaction studies. The method (outlined in Figure 1) is based on optical observation of the dynamics of antibody-functionalized nanorods ('nanoprobes') excited by a rotating magnetic field (RMF). ${ }^{11,12}$ Specifically, the detection relies on nanoprobes with both anisotropic optical and magnetic properties. The most suitable particle type for the measurement method is a cylinder-shaped nanoparticle composed of a ferromagnetic core and a noble metal shell. The latter protects the magnetic core from degradation, while the permanent magnetic moment of the core enables manipulation of its alignment by an external magnetic field. Anisotropic polarizability caused by the elongated nanoprobe geometry leads to optical absorption and scattering factors that strongly depend on the orientation of the nanorods relative to the polarization direction of the incident light, ${ }^{13}$ thus enabling optical detection of the nanoprobes' actual alignment. The nanoprobe's hydrodynamic volume results in a drag torque during its rotational motion, which causes its orientation in the solution to lag behind the momentary direction of the applied RMF by a characteristic phase lag angle. By correlating the momentary 
vector of the applied magnetic field to the measured alignment of the nanoprobes, the phase lag angle of the nanoprobes relative to the applied field direction can be deduced, which is characteristic of the nanoprobe dynamics and increases on target protein binding (see Figure 1 caption for details). ${ }^{11}$ The magnetic excitation mechanism characteristic to our platform enables application of frequency-filtering techniques to the detection signal, which efficiently suppresses interferences originating from complex media and allows us to specifically analyze processes at the nanoprobe surface only. For the experimental realization of the method, see Supporting Section 1.

a) Nanoprobe structure
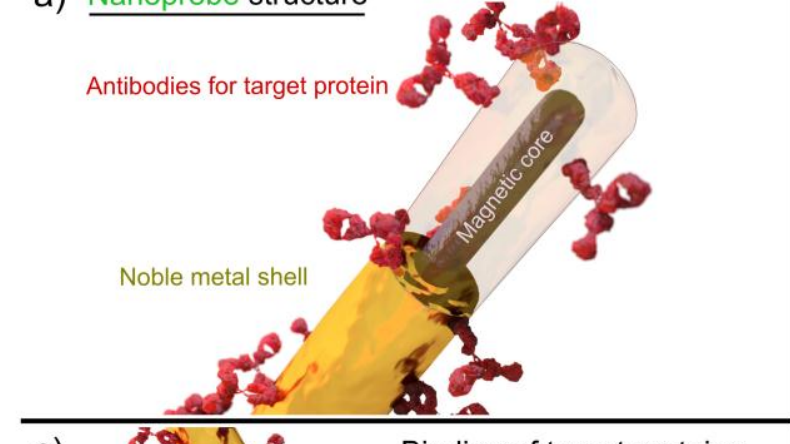

c)

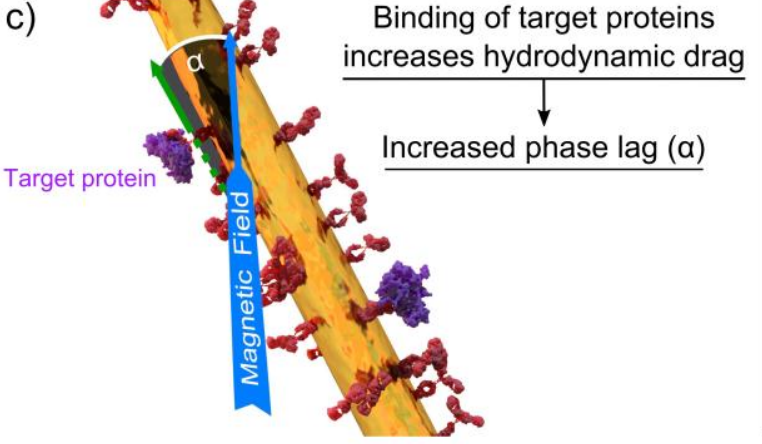

b) Rotating magnetic field rotates nanoprobe

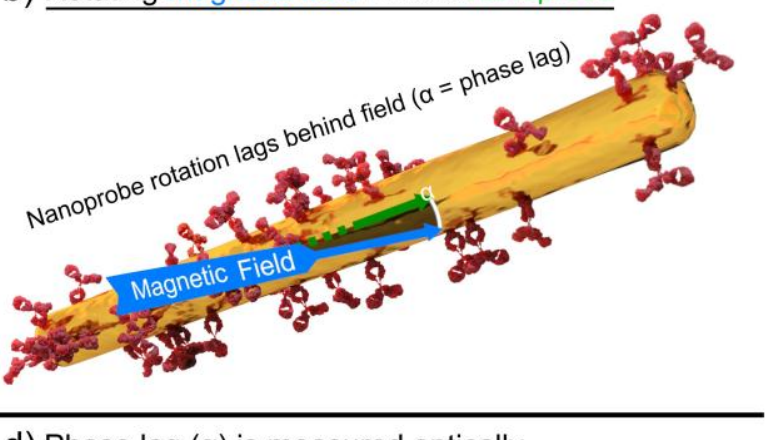

d) Phase lag ( $\alpha$ ) is measured optically

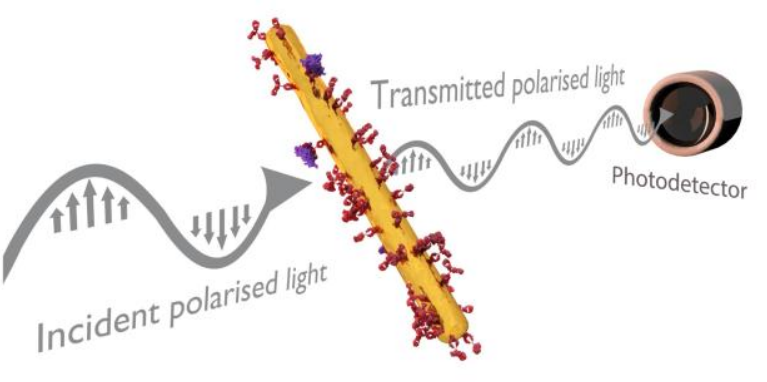

Figure 1. Concept of the measurement procedure. a) Antibody-functionalized magnetic core / noble metal shell nanorods (nanoprobes). b) Nanoprobes are mixed with the sample fluid and excited by a rotating magnetic field (RMF). Due to hydrodynamic drag in the sample fluid, the nanoprobes follow the RMF at a phase lag angle $\alpha$.c) As target molecules bind to the nanoprobes, the hydrodynamic shell thickness and, consequently, $\alpha$ increase. Thus, $\alpha$ represents a direct 
measure of the average number of target molecules bound to the nanoprobes. d) The phase lag angle $\alpha$ is measured optically in transmission geometry using polarized light. The detected intensity depends on the nanoprobe orientation with respect to the polarization direction.

\section{Results and Discussion}

Nanoprobe preparation. While the relative change in nanoprobe dynamics on analyte molecule binding favors small nanorods, overcoming thermal disorder in nanorod alignment requires sufficiently large magnetic moments. ${ }^{11}$ Thus, nanorods comprising a metallic ferromagnetic core are optimal, as their high saturation magnetization allows the application of nanorods with sizes comparable to target proteins. Water stable noble-metal coated cobalt (Co) nanorods were fabricated according to a previously published protocol. ${ }^{14}$ Briefly, plain Co nanorods were synthesized from organometallic precursors in organic solvents to obtain nanorods with excellent size control (see Supporting Figure S2). ${ }^{14,15}$ In subsequent steps, a noble metal shell was synthesized around the core. ${ }^{14}$ Here, sequential deposition of platinum and gold proved to provide the best protection of the core against oxidation (see Supporting Figure S3).

The as-synthesized nanorods are stabilized by hydrophobic ligands in organic solvents. Thus, they need to be transferred to and stabilized in aqueous solutions for antibody functionalization. We developed a water transfer and stabilization procedure that is based on coating the nanorods by an amphiphilic polymer (AP), i.e. a polymer comprising hydrophobic side chains for intercalation with the ligands on the nanorod surface, and a hydrophilic backbone that provides water solubility through charged groups (see Materials and Methods). ${ }^{16}$ The AP-coated waterstabilized nanorods are denoted as 'nanoreagents'.

Following stabilization in water, the nanoreagents were transformed into functional nanoprobes by conjugating antibodies to the carboxy groups of the polymer backbones by carboxy-amine 
linker chemistry (see Materials and Methods). We carried out functionalization of nanoreagents with the monoclonal IgG antibody trastuzumab as recognition agent for the soluble domain of the human epidermal growth factor receptor 2 (sHER2). This antibody has clinical relevance concerning the detection of breast cancer, as well as its treatment. ${ }^{17}$

Protein analysis. To study the nanoprobes' rotational dynamics, we applied a measurement mode that scans the frequency of the external RMF at fixed amplitude ('phase lag spectra'). The measurements were carried out with the nanoprobes immersed in buffer solution at physiological salt conditions (see Materials and Methods). An accurate description of the dynamics of nanorods excited by externally applied RMFs has been achieved by a recently developed theoretical model. ${ }^{18}$ Applying this model, we conducted fits of the measured phase lag spectra, thereby also including actual distributions of nanoparticle parameters. Following an initial fitting procedure to determine basic nanoreagent properties (see Materials and Methods), only the main parameter of interest has been varied for subsequent fits, which is the hydrodynamic shell thickness of the nanoprobes.

Figure $2 \mathrm{a}$ shows the results of the fitting procedure (lines) to measured phase lag spectra (markers) of nanoreagents, of nanoprobes, and of nanoprobes with added sHER2 target protein. Clearly, the phase lag increases with the binding of additional molecular shells, and the fits are in good agreement with the measured data. In order to ensure formation of a homogeneous analyte shell, we applied the analyte molecules in large excess (at least a factor of 150) to the total number of available binding sites. The analyte was added together with a 75-fold higher concentration of bovine serum albumin (BSA) protein, which when added alone did not alter the nanoprobe signal (green markers), thus confirming specific sHER2 binding. By fitting the data obtained for bare nanoprobes (red markers) and for nanoprobes with a fully assembled analyte shell (blue markers), we deduced average shell thicknesses of $15 \pm 9.5 \mathrm{~nm}$ for the antibody functionalization and $25 \pm$ 
$13 \mathrm{~nm}$ for the antibody layer with bound target proteins. These values correlate well with reported IgG antibody ${ }^{19}$ and sHER2 protein $^{20}$ sizes. For target protein analysis, the actual measurement signal is defined by the difference in phase lag angles between bare nanoprobes and nanoprobes with bound analyte. This difference depends on the applied RMF and, for a given amplitude, displays a distinct maximum $\Delta \alpha_{\max }$ at a specific frequency $\mathrm{f}\left(\Delta \alpha_{\max }\right)$. As demonstrated by calculations based on our model and the obtained nanoprobe parameters, $\Delta \alpha_{\max }$ obviously increases with the size of bound target proteins, but the spectral position of the maximum $\mathrm{f}\left(\Delta \alpha_{\max }\right)$ sensitively depends on the target protein size also. In Figure 2b, solid lines correspond to $5 \mathrm{mT}$ RMF amplitude, which has been applied for the protein analysis measurements shown in Figure 2a. Thus, by tuning the excitation of our nanoprobes, we are able to optimize the measurement signal for each target protein size. Therefore, our platform presents a very powerful tool for analyzing protein binding as well as for obtaining their respective sizes.
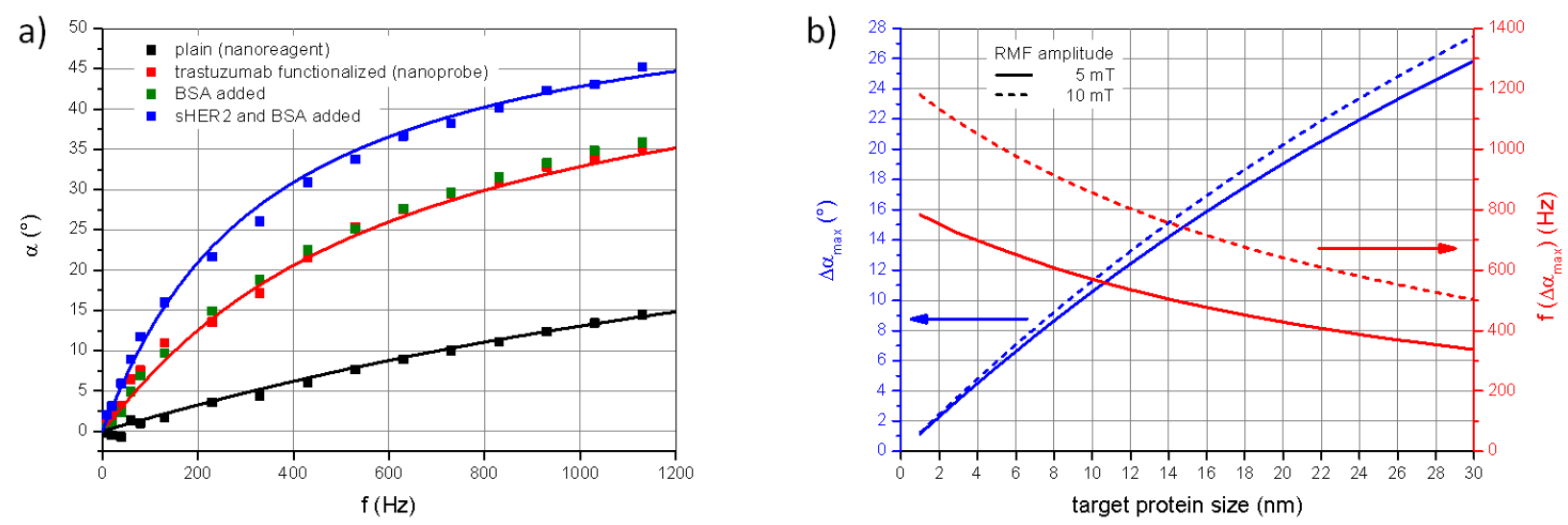

Figure 2. Phase lag spectra and signal dependence on target protein size. a) Measured (markers) and fitted (lines) phase lag angles $\alpha$ of nanoparticles excited by a rotating magnetic field ( $5 \mathrm{mT}$ amplitude) as a function of its frequency $\mathrm{f}$. Phase lag spectra of nanoreagents (black) in comparison to spectra of bare nanoprobes (red) and to nanoprobes with bound analyte shell (blue). Added BSA (green markers) serves as unspecific binding control. b) Calculated magnitude (blue) and spectral 
position (red) of the maximum phase lag difference $\Delta \alpha_{\max }$ upon target protein binding in dependence on the protein size for an applied rotating magnetic field (RMF) amplitude of $5 \mathrm{mT}$ (solid lines) and $10 \mathrm{mT}$ (dashed lines).

Further insights into the dependence of the phase lag difference on different measurement conditions are gained by additional simulations, the results of which are presented in the Supporting Section 3. These include the impact of the initial nanorod aspect ratio on the maximum phase lag difference $\Delta \alpha_{\max }$ and its spectral position $\mathrm{f}\left(\Delta \alpha_{\max }\right)$, which is shown in the Supporting Figure S4. Here, the aspect ratio of the magnetic Co core is varied by varying its length within the experimentally accessible range ${ }^{11}$ from 25 to $180 \mathrm{~nm}$ at a fixed diameter of $5 \mathrm{~nm}$ (see also Supporting Section 2 for the Co core magnetic nanorod characterization). It can be seen that a decrease in initial nanorod size increases $\Delta \alpha_{\max }$ upon target protein binding, which is accompanied by a shift of $\mathrm{f}\left(\Delta \alpha_{\max }\right)$ to higher values. The reason is that the relative increase in hydrodynamic nanoprobe size upon target protein binding and, thus, the measurement signal rises with smaller initial nanorod dimensions. Furthermore, the impact of the magnetic field amplitude and its rotational frequency on the obtained phase lag difference $\Delta \alpha$ is shown in the Supporting Figure S5 in a 3-dimensional plot. Noticeably, for each magnetic field amplitude there is an optimum field frequency at which $\Delta \alpha$ reaches a maximum, ${ }^{12}$ but the maximum value of $\Delta \alpha$ increases with increasing field amplitude, which is attributed to the increasing alignment ratio of the nanoprobes $^{11}$. The dependence of $\Delta \alpha_{\max }$ on the magnetic field amplitude, however, is rather weak. Thus, the experimental realization of a suitable measurement setup is based on a compromise of high magnetic field strengths and technical realizability. Finally, in Figure S6, we demonstrate the impact on the standard deviations of the hydrodynamic protein shells on the measurement signal. Specifically, $\Delta \alpha_{\max }$ and $\mathrm{f}\left(\Delta \alpha_{\max }\right)$ with the actual protein shell standard deviations are compared to 
respective results for assumed nanoprobes with zero as well as halved and doubled protein layer thickness standard deviations. As expected, a decrease in the protein shell thickness standard deviations increases the obtainable phase lag differences. At the same time, $f\left(\Delta \alpha_{\max }\right)$ increases with decreasing protein layer thickness standard deviations.

Signal dependence on analyte protein concentration. To obtain the dependence of the measurement signal on the concentration of analyte molecules present in the solution, it is sufficient to measure at a single frequency of the RMF and determine the relative phase lag difference with respect to a reference. To that end, we employed an adapted measurement setup that is capable of applying a higher RMF amplitude of $10 \mathrm{mT}$ at a fixed frequency of $1 \mathrm{kHz}$ (see Supporting Section 1), which was chosen for optimal measurement signal for usually encountered analyte protein sizes of about 2-15 nm (see red dashed line in Figure $2 b$ ). While the higher field amplitude also leads to a slightly larger phase lag difference $\Delta \alpha_{\max }$ as compared to $5 \mathrm{mT}$ amplitude (see blue lines in Figure 2b), the main advantage is the resulting increase in the optical signal (by a factor of 2.7 ) due to the higher alignment ratio of the nanoprobes. The measured signal dependence on analyte protein concentration is shown in Figure 3. The error bars correspond to the error that follows by the error propagation law including the standard deviations of the reference and the respective analyte-spiked samples. All measurements are carried out directly after mixing the nanoprobes with the analyte molecules, at which time the phase lag signal is already stationary, which demonstrates the rapidity of our homogeneous platform. Clearly, due to an increasing hydrodynamic volume of the nanorods caused by a rising binding density of sHER 2 proteins to the immobilized trastuzumab antibodies, the signal $\Delta \alpha$ increases with rising analyte concentration. To determine the assay performance of our platform, we applied a logistic fit. ${ }^{21}$ According to these results (see Figure 3), we are able to detect sHER2 protein for concentrations 
higher than $0.44 \mathrm{nM}$ (limit of detection, i.e. the sHER2 concentration at which the signal reaches $3 \mathrm{x}$ the error of the $1 \mathrm{nM}$ sHER2 sample).

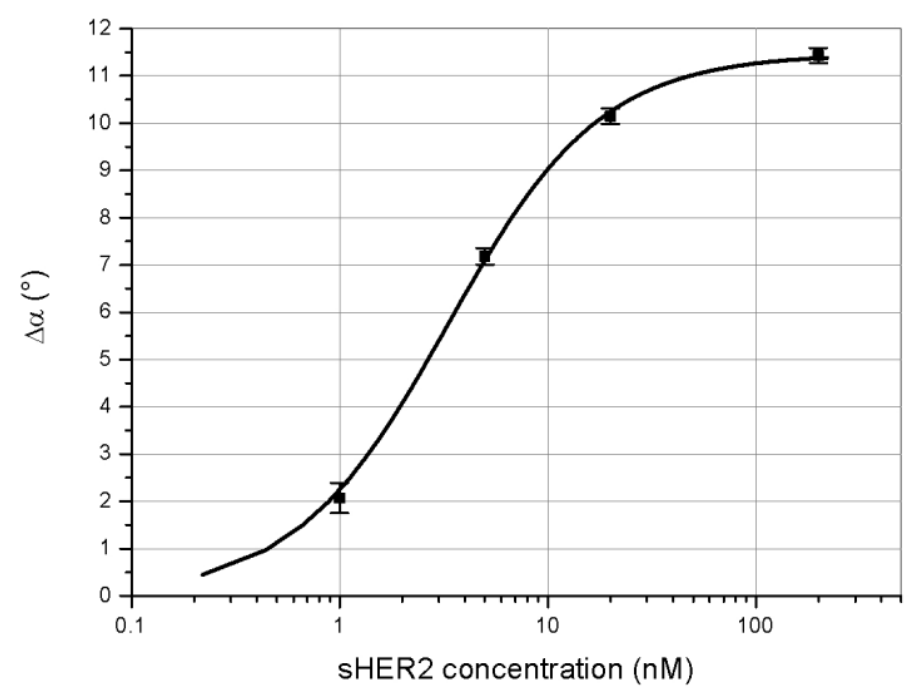

Figure 3. Signal dependence on the analyte concentration. Measured nanoprobe phase difference $\Delta \alpha$ (markers) and logistic fit (line) in dependence of the concentration of spiked sHER2 with respect to a reference sample without sHER2. Nanoprobes are excited by a rotating magnetic field of $10 \mathrm{mT}$ amplitude at a frequency of $1000 \mathrm{~Hz}$.

Nanoprobe dispersion stability. The parameters resulting from our model fits agree well to a single particle dispersion state of the nanoprobes. Our measurement method relies on stable nanoprobe dispersions, which for ferromagnetic nanoparticles is generally difficult to achieve due to the strong magnetic interparticle attraction. ${ }^{22}$ Thus, we examined stability criteria by carrying out distance-dependent total interaction energy calculations for nanorods aligned side-by-side, which is the preferred assembly of the nanorods independent of solvent-specific interactions ${ }^{15}$ (see also Supporting Section 4). For stable nanoparticle dispersions, the attractive magnetic and van der Waals interactions need to be counterbalanced by sufficiently strong repulsive forces. ${ }^{23}$ While in low ionic strength solvents, such stability can be obtained by electrostatic repulsion (see Supporting Figure S9 for nanoreagent stock solutions in water), the free ions present in biological 
buffers effectively screen electrostatic interactions. ${ }^{23}$ However, the hydration interaction caused by rearrangement of solvent molecules in-between two approaching surfaces has been shown to increase linearly with the solvent's ionic strength, ${ }^{24}$ which, as we show below, provides sufficient repulsive interaction for stable single particle dispersions in buffer.

Figure 4 shows the calculated distance-dependent total interaction energies for the most relevant nanorod type for our measurement platform, i.e. bare nanoprobes (additional nanorod types are discussed in Supporting Section 4). As the solvent in this case is a high ionic strength buffer, the only included repulsive force is the hydration interaction. The explicit formulas and parameters employed for the interaction energy calculations are detailed in Supporting Section 4. While all other relevant parameters are deduced unambiguously from experimental data or literature values, the reported parameters governing the hydration interaction (strength and decay length) do vary within certain ranges. ${ }^{23}$ Consequently, the resulting interaction energy also varies, which is represented in the graphs by the grey-shaded area. Looking at the most unfavorable case of weakest hydration repulsion (lower border of the grey-shaded region), the total interaction energy of two approaching nanoprobes shows a shallow potential well at a separation of about 3 - $4 \mathrm{~nm}$, followed by a large potential barrier to shorter distances. For well-dispersed nanoparticles, the potential barrier needs to be sufficiently high to prevent irreversible agglomeration, while the depth of the potential well has to be low enough to avoid metastable nanoparticle assemblies. We deduce a minimally required energy barrier of $25 k_{B} T$ to achieve stable nanoprobe dispersions (see Supporting Section 4f). As the calculated energy barrier for the least favorable case still amounts to about $100 k_{B} T$, irreversible nanoprobe agglomeration can be excluded. To evaluate the effect of the potential well with a maximum depth of about $3.2 k_{B} T$, we transferred the calculated interaction energy into a linear particle probability density, from which we approximate that about $97 \%$ of 
the nanoprobes are free to rotate without interference by another nanoprobe (see Supporting Section 4g). Thus, despite our nanoprobes being ferromagnetic, they form well-dispersed suspensions and are fully applicable to homogeneous protein analysis
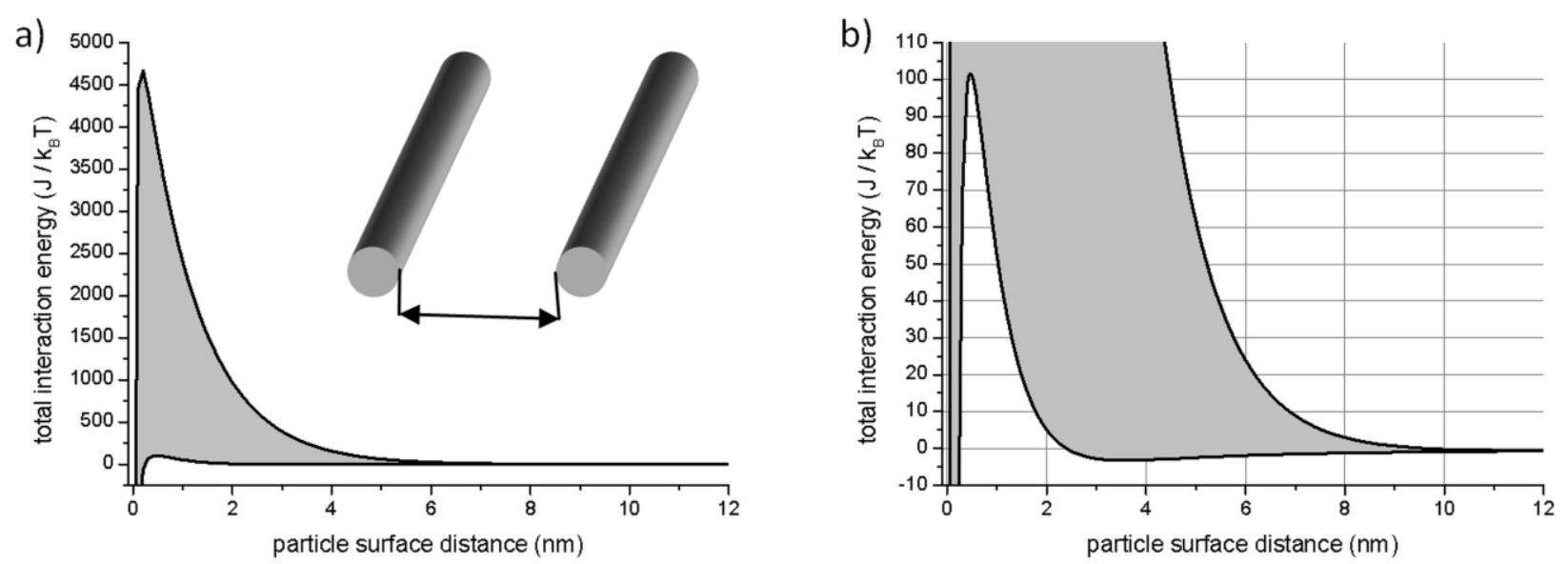

Figure 4. Stability of nanoprobes in buffer solution. a) Calculated total particle interaction energies in dependence of the particle surface distance. Nanoprobes are assembled side by side with the particle surface distance taken between their antibody shells. The grey-shaded area represents the influence of variations of the hydration interaction parameters (strength and decay length) onto the total interaction energy. b) Zoom of the energy barrier with a height of about $100 k_{B} T$ for weakest total interaction.

\section{Conclusions and Outlook}

Our results show that we can turn ferromagnetic core-shell nanorods into stable functional nanoprobes for specific solution-based investigation of protein binding. We demonstrate that our platform is applicable to direct protein size analysis, which shows great promise for studying molecular dynamics and interactions at the nanoprobe surface. In principle, our platform can be applied to every reaction that alters the nanoprobes' rotational dynamics, such as research on protein-protein and protein-DNA interactions. Possible studies include protein oligomerization, ${ }^{25}$ 
helicase and translocase activities ${ }^{2}$ as well as transcription factors involved in DNA looping processes. $^{26}$

Apart from fundamental protein interaction studies, our platform is also promising for in-vitro biomarker testing for molecular diagnostic applications. We demonstrate a detection limit of 440 pM for sHER2 protein by our simple solution-based mix \& measure approach. This value can be chosen for a first comparison to a commercially available central laboratory analyzer, which is the Siemens ADVIA Centaur ${ }^{\circledR}$ system based on chemiluminescence detection of the sHER2 target protein in a sandwich immunoassay format. The sensitivity of the ADVIA Centaur ${ }^{\circledR}$ system spans well below the clinical cut-off sHER2 concentration of $170 \mathrm{pM}^{27}$, but its technical realization is not compatible with the ease of handling requirements for point-of-care testing, which can be met by our homogeneous mix \& measure platform following further technological refinements. Extension to multiplex detection could be achieved in a straightforward way by making use of differently functionalized nanorod batches dispersed in separate chambers of a fluidic cartridge which are measured sequentially.

\section{Materials and Methods}

Polymer stabilization. We applied an amphiphilic polymer (polymer backbone of poly(isobutylene-alt-maleic anhydride) with hydrophobic dodecylamine side chains, henceforth abbreviated by AP) at a concentration of $0.5 \mathrm{M}$ in chloroform. ${ }^{16} \mathrm{Next}$, the noble metal shell coated nanorods dispersed in toluene at a concentration of $120 \mathrm{nM}$ were diluted by tetrahydrofuran (THF) and by addition of the AP (5200 monomers of AP per $\mathrm{nm}^{2}$ of particle surface) to a total volume of $\sim 2 \mathrm{ml}$. This solution was vaporized in a rotating evaporator at $600 \mathrm{mbar}$ and $70{ }^{\circ} \mathrm{C}$ for approximately $30 \mathrm{~min}$. The dried polymer-particle solution was re-dissolved in $\sim 2 \mathrm{ml}$ of $\mathrm{THF}$, and vaporized for another $15 \mathrm{~min}$ to ensure that the AP is well wrapped around the nanorods. Next, the 
dried solution was re-dissolved in $\sim 6 \mathrm{ml}$ of $100 \mathrm{mM} \mathrm{NaOH}$ to hydrolyze the polymer backbone. Sonication of about $5 \mathrm{~min}$ at $120 \mathrm{~W}$ was needed to completely solubilize all particles. Washing of the AP-coated nanorods with milli-Q water was done by repetitive $(3 \mathrm{x})$ precipitation in a centrifuge. Final particle concentrations of $13 \mathrm{nM}$ were obtained (see Supporting Section 2 for particle concentration determination).

Nanoparticle functionalization. For binding of monoclonal trastuzumab antibodies, the carboxy groups on the AP backbone were activated to covalently bind amine groups present on the antibody. The functionalization reaction was carried out in 2-(N-morpholino)ethanesulfonic acid (MES) buffer solution at a $\mathrm{pH}$ value of 5.5. Under these conditions, the antibodies will be adsorbed preferentially with the Fc/central region, promoting the reaction of the amine groups located in this part with the nanoreagents, thus, favoring oriented binding. ${ }^{28}$ AP coated nanorods dissolved in water at a particle concentration of $13 \mathrm{nM}$ were incubated with EDC (N-(3Dimethylaminopropyl)-N'-ethylcarbodiimide hydrochloride) and S-NHS (NHydroxysulfosuccinimide sodium salt) for $20 \mathrm{~min}$ at room temperature. Both EDC and S-NHS molecules were suspended in a solution of $50 \mathrm{mM}$ MES buffer and $\sim 3.3 \cdot 10^{5}$ EDC molecules and $\sim 9.9 \cdot 10^{5}$ S-NHS molecules were used per nanorod. The final MES concentration amounted to 2 $\mathrm{mM}$. Then, the trastuzumab antibodies ( $\sim 50$ antibodies per nanorod) were added, and the solution was incubated for $120 \mathrm{~min}$ at $4{ }^{\circ} \mathrm{C}$. Afterwards, BSA (bovine serum albumin) was added to block any remaining binding sites, and another incubation step at $4{ }^{\circ} \mathrm{C}$ was carried out overnight with an excess of $\sim 1100$ BSA molecules per nanorod. The sample was washed with PBS buffer (150 mM sodium phosphate, $150 \mathrm{mM} \mathrm{NaCl}$ at a $\mathrm{pH}$ of 7.4) by precipitation in the centrifuge (Eppendorf MiniSpin, $8000 \mathrm{rpm}, 10 \mathrm{~min}$ ). Re-dispersion was carried out by vortexing and mild sonication for 3 s (VWR ultrasonic cleaner USC500D, power level 1). Washing and re-dispersion was done 3x 
and resulting samples were stored at $4{ }^{\circ} \mathrm{C}$. Final functionalized particle concentrations of $1.4 \mathrm{nM}$ were obtained (see Supporting Section 2 for particle concentration determination).

For a second batch of functionalized nanorods, the blocking step of the functionalization procedure was modified. Ethanolamine instead of BSA was used in an excess of $\sim 3.3 \cdot 10^{7}$ molecules per nanorod. Blocking with ethanolamine was done for $90 \mathrm{~min}$ at $4{ }^{\circ} \mathrm{C}$. Washing was done according to the procedure described above except that instead of PBS, the measurement buffer solution (TBS, see paragraph below) was employed for re-dispersion. Final functionalized particle concentrations of $54 \mathrm{pM}$ were obtained (see Supporting Section 2 for particle concentration determination).

Measurement procedure. Protein analysis (phase lag spectra) was carried out in TBS buffer solution (25 mM Tris, $150 \mathrm{mM} \mathrm{NaCl}, \mathrm{pH}=7.4)$ at a nanoreagent / nanoprobe concentration of 32 pM / $13 \mathrm{pM}$ (see Supporting Section 2 for particle concentration determination). The sHER2 analyte (recombinant protein) was spiked to the sample solution containing nanoprobes to yield a defined sHER2 concentration of $200 \mathrm{nM}$.

The signal dependence on the analyte protein concentration was carried out in TBS buffer at a nanoprobe concentration of $3 \mathrm{pM}$ (see Supporting Section 2 for particle concentration determination). The sHER2 analyte (recombinant protein) was spiked to the sample solution containing nanoprobes to yield defined sHER2 concentrations of 1, 5, 20 and $200 \mathrm{nM}$.

All measurements were started within 5 min after mixing the sample solutions.

Data fitting procedure for protein analysis. The applied theoretical model is based on a set of empirical equations derived from numerical solutions of the Fokker-Planck equation for determining the dynamic response of magnetic nanoparticles excited by RMFs. ${ }^{18}$ For fitting the recorded phase lag spectra in dependence on the frequency of the externally applied RMF, input 
parameters comprised the RMF characteristics, the nanoparticle parameters and the viscosity and temperature of the solvent. Average particle geometry parameters for the fit were independently derived by TEM image analysis of plain Co nanorods. The average magnetic moment per nanorod was deduced from the magnetic volume and the Co saturation magnetization, which for our Co nanorods can be assumed to be identical to the bulk Co value ${ }^{29}$. In a first fitting step, the hydrodynamic shell thickness after the polymer coating was determined. Here, the average value and standard deviation (Gaussian distribution) of the hydrodynamic shell thickness as well as the standard deviation of the magnetic moment were the free fit parameters. This procedure resulted in a first hydrodynamic shell of $5.5 \mathrm{~nm}$ thickness, which includes the noble metal shell layer $(\sim 1.9$ $\mathrm{nm}$, see Supporting Section 2$)$, the ligand shell $\left(\sim 2 \mathrm{~nm}\right.$ according to the hexadecylamine ${ }^{14}$ molecular structure and the mean carbon-carbon bond length ${ }^{30}$ ) and the coated polymer, which also includes the stagnant surface layer formed in the particle-dispensing solution. Specifically, the fitting resulted in a magnetic moment of $1.5 \pm 0.85 \cdot 10^{-18} \mathrm{Am}^{2}$, a nanoreagent length of $63 \pm$ $45 \mathrm{~nm}$ and a diameter of $16 \pm 6 \mathrm{~nm}$. Based on the so obtained parameters, the fitting of the antibody functionalized particles (nanoprobes) and of the antigen coated nanoprobes was performed. For these fits, only the hydrodynamic shell parameters were varied.

\title{
ASSOCIATED CONTENT
}

Supporting Information. Details of the experimental measurement setup; nanorod characterization; particle stability calculations

This material is available free of charge via the Internet at http://pubs.acs.org.

\author{
AUTHOR INFORMATION
}




\title{
Corresponding Author \\ *E-mail: Stefan.Schrittwieser@ait.ac.at
}

\author{
Present Addresses \\ $\dagger^{\dagger}$ Biological and Environmental Sciences and Engineering Division, King Abdullah University of \\ Science and Technology (KAUST), Thuwal, Kingdom of Saudi Arabia \\ Diagnostic and Interventional Radiology Department and Clinic, Universitätsklinikum Hamburg- \\ Eppendorf(UKE), Hamburg, Germany
}

\section{Notes}

The authors declare no competing financial interest.

\section{ACKNOWLEDGMENT}

The authors thank Frauke Alves, Julia Bode and Fernanda Ramos Gomes from the Max-PlanckInstitute of Experimental Medicine in Göttingen for providing the trastuzumab antibody in form of the Herceptin therapeutic drug. The figure showing the measurement principle has been created by Darragh Crotty (www.darraghcrotty.com). Parts of this research were supported by the European Commission FP7 NAMDIATREAM project (EU NMP4-LA-2010-246479), by the German research foundation (DFG grant GRK 1782 to W.J.P.), and by the European Research Council (ERC Starting Grant \#335078 Colouratom). B.P. acknowledges a PostDoctoral fellowship from the Alexander von Humboldt foundation.

\section{REFERENCES}

(1) Fehon, R. G.; McClatchey, A. I.; Bretscher, A. Organizing the Cell Cortex: the Role of ERM Proteins. Nat. Rev. Mol. Cell Biol. 2010, 11, 276-287. 
(2) Lohman, T. M.; Tomko, E. J.; Wu, C. G. Non-Hexameric DNA Helicases and Ttranslocases: Mechanisms and Regulation. Nat. Rev. Mol. Cell Biol. 2008, 9, 391-401.

(3) Kristiansen, G. Diagnostic and Prognostic Molecular Biomarkers for Prostate Cancer. Histopathology 2012, 60, 125-141.

(4) Pearson, T. A. Markers of Inflammation and Cardiovascular Disease: Application to Clinical and Public Health Practice: a Statement for Healthcare Professionals from the Centers for Disease Control and Prevention and the American Heart Association. Circulation 2003, 107, 499-511.

(5) Ries, J.; Schwille, P. Fluorescence Correlation Spectroscopy. Bioessays 2012, 34, 361-368.

(6) Jameson, D. M.; Ross, J. A. Fluorescence Polarization/Anisotropy in Diagnostics and Imaging. Chem. Rev. 2010, 110, 2685-2708.

(7) Wienken, C. J.; Baaske, P.; Rothbauer, U.; Braun, D.; Duhr, S. Protein-Binding Assays in Biological Liquids Using Microscale Thermophoresis. Nat. Commun. 2010, 1, 100.

(8) Zhang, H.; Li, F.; Dever, B.; Li, X.-F.; Le, X. C. DNA-Mediated Homogeneous Binding Assays for Nucleic Acids and Proteins. Chem. Rev. 2013, 113, 2812-2841.

(9) Ruedas-Rama, M. J.; Walters, J. D.; Orte, A.; Hall, Elizabeth A H. Fluorescent Nanoparticles for Intracellular Sensing: a Review. Anal. Chim. Acta 2012, 751, 1-23.

(10) Lee, D.-E.; Koo, H.; Sun, I.-C.; Ryu, J. H.; Kim, K.; Kwon, I. C. Multifunctional Nanoparticles for Multimodal Imaging and Theragnosis. Chem. Soc. Rev. 2012, 41, 2656-2672.

(11) Schrittwieser, S.; Ludwig, F.; Dieckhoff, J.; Soulantica, K.; Viau, G.; Lacroix, L.-M.; Lentijo, S. M.; Boubekri, R.; Maynadié, J.; Huetten, A.; Brueckl, H.; Schotter, J. Modeling and 
Development of a Biosensor Based on Optical Relaxation Measurements of Hybrid Nanoparticles. ACS Nano 2012, 6, 791-801.

(12) Schrittwieser, S.; Ludwig, F.; Dieckhoff, J.; Tschoepe, A.; Guenther, A.; Richter, M.; Huetten, A.; Brueckl, H.; Schotter, J. Direct Protein Detection in the Sample Solution by Monitoring Rotational Dynamics of Nickel Nanorods. Small 2014, 10, 407-411.

(13) Bohren, C. F.; Huffman, D. R. Absorption and Scattering of Light by Small Particles, Wiley: New York, 1983.

(14) Lentijo-Mozo, S.; Tan, R. P.; Garcia-Marcelot, C.; Altantzis, T.; Fazzini, P.-F.; Hungria, T.; Cormary, B.; Gallagher, J. R.; Miller, J. T.; Martinez, H.; Schrittwieser, S.; Schotter, J.; Respaud, M.; Bals, S.; van Tendeloo, G.; Gatel, C.; Soulantica, K. Air- and Water-Resistant Noble Metal Coated Ferromagnetic Cobalt Nanorods. ACS Nano 2015, 9, 2792-2804.

(15) Wetz, F.; Soulantica, K.; Respaud, M.; Falqui, A.; Chaudret, B. Synthesis and Magnetic Properties of Co Nanorod Superlattices. Mater. Sci. Eng., C 2007, 27, 1162-1166.

(16) Soliman, M. G.; Pelaz, B.; Parak, W. J.; Del Pino, P. Phase Transfer and Polymer Coating Methods Toward Improving the Stability of Metallic Nanoparticles for Biological Applications. Chem. Mater. 2015, 27, 990-997.

(17) Molina, R.; Barak, V.; van Dalen, A.; Duffy, M. J.; Einarsson, R.; Gion, M.; Goike, H.; Lamerz, R.; Nap, M.; Sölétormos, G.; Stieber, P. Tumor Markers in Breast Cancer - European Group on Tumor Markers Recommendations. Tumour Biol. 2005, 26, 281-293.

(18) Yoshida, T.; Enpuku, K.; Dieckhoff, J.; Schilling, M.; Ludwig, F. Magnetic Fluid Dynamics in a Rotating Magnetic Field. J. Appl. Phys. 2012, 111, 053901. 
(19) Jung, Y.; Jeong, J. Y.; Chung, B. H. Recent Advances in Immobilization Methods of Antibodies on Solid Supports. Analyst 2008, 133, 697-701.

(20) Vicente-Alique, E.; Núñez-Ramírez, R.; Vega, J. F.; Hu, P.; Martínez-Salazar, J. Size and Conformational Features of ErbB2 and ErbB3 Receptors: a TEM and DLS Comparative Study. Eur. Biophys. J. 2011, 40, 835-842.

(21) Chieh, J. J.; Yang, S.-Y.; Horng, H.-E.; Yu, C. Y.; Lee, C. L.; Wu, H. L.; Hong, C.-Y.; Yang, H.-C. Immunomagnetic Reduction Assay Using High-Tc Superconducting-QuantumInterference-Device-Based Magnetosusceptometry. J. Appl. Phys. 2010, 107, 074903.

(22) Bishop, Kyle J M; Wilmer, C. E.; Soh, S.; Grzybowski, B. A. Nanoscale Forces and Their Uses in Self-Assembly. Small 2009, 5, 1600-1630.

(23) Israelachvili, J. N. Intermolecular and Surface Forces, 3rd ed, Academic Press: Burlington, MA, 2011.

(24) Molina-Bolívar, J. A.; Galisteo-González, F.; Hidalgo-Álvarez, R. Colloidal Stability of Protein-Polymer Systems: A Possible Explanation by Hydration Forces. Phys. Rev. E 1997, 55, $4522-4530$.

(25) Eisenberg, D.; Jucker, M. The Amyloid State of Proteins in Human Diseases. Cell 2012, $148,1188-1203$.

(26) Marenduzzo, D.; Faro-Trindade, I.; Cook, P. R. What are the Molecular Ties that Maintain Genomic Loops? Trends Genet. 2007, 23, 126-133. 
(27) Tsé, C.; Gauchez, A.-S.; Jacot, W.; Lamy, P.-J. HER2 shedding and serum HER2 extracellular domain: Biology and clinical utility in breast cancer. Cancer Treat. Rev. 2012, 38, $133-142$.

(28) Puertas, S.; Batalla, P.; Moros, M.; Polo, E.; Del Pino, P.; Guisan, J. M.; Grazú, V.; de la Fuente, Jesús M. Taking Advantage of Unspecific Interactions to Produce Highly Active Magnetic Nanoparticle-Antibody Conjugates. ACS Nano 2011, 5, 4521-4528.

(29) Soulantica, K.; Wetz, F.; Maynadié, J.; Falqui, A.; Tan, R. P.; Blon, T.; Chaudret, B.; Respaud, M. Magnetism of Single-Crystalline Co Nanorods. Appl. Phys. Lett. 2009, 95, 152504.

(30) Bartell, L. S. On the Length of the Carbon-Carbon Single Bond. J. Am. Chem. Soc. 1959, $81,3497-3498$. 
Table of Contents Graphic

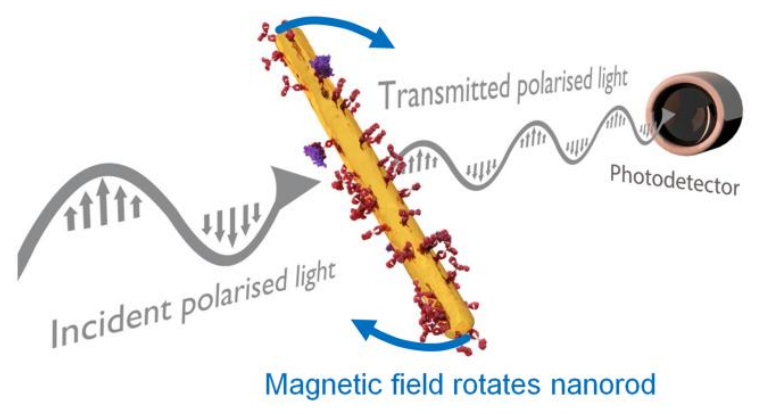

20

21

22

23

24

25

26

27

28

29

30

31

32

33

34

35

36

37

38

39

40

41

42

43

44

45

46

47

48

49

50

51

52

53

54

55

56

57

58

59

60

ACS Paragon Plus Environment 